\title{
El campo de la comunicación y la cultura como reflejo y agencia en lberoamérica
}

The field of communication and culture as reflection and agency in Ibero-America

Martín Becerra, Universidad Nacional de Quilmes y Universidad de Buenos Aires - mbecerra@unq.edu.ar

\section{Resumen}

El presente artículo analiza la reconfiguración de la cooperación iberoamericana en comunicación y cultura desde los ángulos de la estructuración de los sistemas infocomunicacionales, su producción cultural y su agenda de estudios académicos y científicos propios del campo disciplinar. El texto propone un abordaje exploratorio sobre un proceso que registra distintas etapas en materia de cooperación y que toma como referencia la evolución material del sector industrial en comunicación y cultura, las políticas públicas concertadas en el espacio iberoamericano y la constitución y desarrollo del campo académico, tomándolo como reflejo de las condiciones estructurales y como agencia de problemas y agenda de reflexión y debate.

\section{Palabras clave}

Iberoamérica, cooperación, comunicación, cultura, estructura, infocomunicación, Latinoamérica.

\section{Abstract}

This article analyses the reconfiguration of Ibero-American cooperation in communication and culture from the angles of the structuring of the info-communication systems, its cultural production and its agenda of their own academic and scientific studies of the discipline. The text proposes an exploratory approach on a process that registers different stages in the field of cooperation and that takes as reference the material evolution of the industrial sector in communication and culture, concerted public policies in the lbero-American area and the establishment and development of the academic field, taking it as a reflection of structural conditions and as an agency of problems and an agenda for reflection and debate.

\section{Keywords}

Ibero-America, cooperation, communication, culture, structure, infocommunication, Latin America.

Sumario

1. La comunicación como escena material. 2. El campo académico: ¿por casa cómo andamos? 3. A modo de conclusión: reflejo y agencia. 4. Referencias bibliográficas. 


\section{La comunicación como escena material}

Iberoamérica es un espacio que desde hace 50 años produce reflexión sobre la configuración de los sectores de información, comunicación y cultura, con tradiciones que fueron señeras, pero en la última década parte de ese acervo acusa un proceso de dispersión que es analizado, mediante un abordaje exploratorio, en el presente artículo. Las premisas del trabajo están contenidas en dos paradojas en el ámbito de la comunicación, la cultura y la cooperación. La primera paradoja es que mientras la globalización informacional representa una pulsión hacia la articulación e integración de flujos culturales y comunicativos, al finalizar la segunda década del siglo XXI el espacio iberoamericano registra tendencias centrífugas en sus relaciones de cooperación en el marco del propio espacio en pos de un mayor acercamiento, por separado, con potencias tradicionales (Estados Unidos) o emergentes (China). La segunda paradoja es que mientras que la producción industrial y masiva de información y cultura en Iberoamérica es especialmente dependiente de la intervención y el financiamiento públicos, incluso cuando su gestión y explotación es cedida al sector privado comercial, los ejemplos más estables de estrategias y prácticas de cooperación han sido tutelados por una lógica de mercantilización y administración privada o, en el mejor de los casos, mixta.

A modo de introducción, corresponde en primer lugar señalar que como toda clasificación la de lberoamérica ameritaría una reflexión acerca de su pertinencia, ya que agrupa a dos países europeos (España y Portugal) con al menos 20 países latinoamericanos, cuya delimitación a su vez es variable, pues no siempre se incluye a enclaves franceses en el Caribe o a Puerto Rico, estado asociado a Estados Unidos de América, por ejemplo. En este sentido, resulta adecuada la advertencia de Miquel de Morgas Spà cuando en su libro "Interpretar la comunicación" (2011) considera inadecuado comparar la investigación académica de un solo país (España) con una región vasta e integrada por numerosos países con tradiciones no siempre coincidentes como América Latina y, por ello, propone ampliar el estudio a la Europa Latina (España, Francia, Italia y Portugal) para proceder a su contraste con Latinoamérica. No obstante, la definición del contorno geográfico y cultural del presente número se centra específicamente en Iberoamérica y, por consiguiente, las siguientes páginas harán referencia a este espacio. A continuación, uno de los desafíos conceptuales consiste en definir qué tan apropiado resulta el término "región", que tiene diferentes acepciones, para su aplicación al espacio iberoamericano. Dado que su el uso del concepto "región" es dispar, polisémico y en algunos países incluso controvertido (ver Camacho Chavez, 2016), en el presente trabajo se alude a Iberoamérica como espacio.

Tanto desde la perspectiva de la estructuración de las actividades infocomunicacionales como desde la producción cultural, Iberoamérica tuvo en el Quinto Centenario del arribo de Colón a América un hito que intensificó la creación de programas de cooperación y su institucionalización así como activó, además, los vínculos y producciones en una escala desconocida hasta ese momento. España fue durante la década de 1990 y los primeros años de este siglo el animador central de esos programas, políticas y prácticas, y lo fue desde dos niveles que analíticamente corresponde diferenciar: en primer lugar, desde sus gobiernos con financiación pública y promoción de acuerdos y coproducciones culturales que en general eran bilaterales (es decir, de España con algún país latinoamericano) y no multilaterales (es decir, entre varios países del espacio iberoamericano); en segundo lugar, desde el capital privado a través del protagonismo que cobraron corporaciones del sector industrial de los medios y las telecomunicaciones (como Telefónica y el Grupo PRISA) o del sector financiero (como los bancos Santander y BBVA), beneficiados por las políticas de privatizaciones llevadas a cabo por los gobiernos latinoamericanos en ese lapso. El escenario de la cooperación iberoamericana vivió entonces una suerte de edad dorada que repercutió, también, en el campo académico.

Sin embargo, en la última década aquellos bríos se apaciguaron con cambios de estrategia en las políticas públicas de los diferentes países, en particular las de España como principal financiador, y en la medida que los ecos noventistas se fueron apagando, la asignación de prioridades en materia de cooperación en comunicación y cultura dejó de tener como espacio de referencia principal a lberoamérica.

Los giros que dieron las políticas gubernamentales en numerosos países del ámbito iberoamericano en relación a la cooperación en las últimas décadas ponen en evidencia, como señaló Bustamante (2007), "la ausencia de auténticas concepciones de Estado sobre la lengua y la cultura y, por tanto, sobre su valor de proyección exterior". Con la evidencia de la última década se puede complementar el planteo de Bustamante y constatar que esa falta de concepciones de Estado en relación a la cultura está subordinada a estrategias más generales de procesos de integración económica (como los tratados bilaterales de "libre" comercio suscriptos por países latinoamericanos y EEUU, por ejemplo) o políticoeconómica (como la apuesta por centrarse en la participación y construcción de la Unión Europea en los casos de España y Portugal). El cambio de prioridades en política exterior de muchos de los países que protagonizaron el auge de la cooperación en lberoamérica entre 1990 y 2005 expresa un giro que tuvo efectos materiales concretos tanto en el posicionamiento de cada uno de ellos en la escena internacional, como también en la redefinición del valor relativo de la integración iberoamericana y, consecuentemente, en la producción conjunta de cultura y comunicación.

En efecto, fruto de ese giro en la última década los países con mayores capacidades y recursos (que no siempre van de la mano) en América Latina tienen diferentes interlocutores y ello ha redireccionado sus políticas de cooperación (México, Colombia, Perú y Chile con EEUU; Brasil con EEUU, China y parte de Europa; Argentina con EEUU y China); es decir, ya no hay una vía preferencial con España/Portugal.

En palabras de Camacho Chavez, quien realizó una tesis de Maestría sobre la cooperación iberoamericana en el sector audiovisual, "a los cambios en la balanza de poder regional y una estrategia de liderazgo poco acertado de parte de España, se debe sumar la duplicidad de roles de España entre Latinoamérica y la Unión Europea. Como se citó anteriormente, España ha jugado a ser un puente natural entre las dos regiones, de esta forma, el esquema regional iberoamericano se construyó bajo el ideal de su fuerte potencial para proyectar internacionalmente a América Latina y mejorar su diálogo con Europa. Sin embargo, como explica Belén Blázquez (2014) los países iberoamericanos no perciben que hoy sea necesario el puente español para representar sus intereses en Europa en distintos ámbitos de cooperación" (Camacho Chavez, 2016).

Del proyecto de un espacio común iberoamericano que funcionara como dinamizador y activador no sólo colectivo sino también como capital de cada uno de sus integrantes, se percibe el desplazamiento a un universo en expansión donde los puntos otrora cercanos se alejan entre sí a la vez que muchos de ellos adquieren mayor gravitación relativa en su contacto con países ajenos a ese proyecto. En las casi tres décadas que comprende el lapso desde 1990 hasta el presente, además, todos los países de lberoamérica padecieron graves crisis económicas, sociales (y, en 
muchos casos, también institucionales) y tramitaron de modo muy heterogéneo la salida a las mismas; es decir que la salida a las crisis tampoco representó un ejercicio común o compartido, ni lo es, pues, su saldo social.

Si bien las razones de los movimientos y cambios de sentido de las estrategias de cooperación iberoamericana en las últimas décadas son fundamentalmente políticas y económicas, es posible advertir que el sector incofomunicacional en particular ha tenido incidencia en este proceso. En efecto, las crisis orgánicas que protagonizaron los distintos países de la región y la emergencia de un nuevo ecosistema en las comunicaciones, fundamentalmente signado por la cultura de la convergencia, su correlato de desprogramación y de la llamada "autocomunicación de masas" (Castells, 2009), la emergencia de nuevos intermediadores globales de la circulación de contenidos, la diseminación de las tecnologías digitales y la consecuente desintermediación de la labor de los medios tradicionales son factores que también potenciaron la lógica centrífuga de la cooperación iberoamericana.

La globalización y la convergencia no son procesos con tendencias únicamente uniformizadoras en lo cultural, sino también fragmentadoras y contradictorias, como sostienen García Canclini (2004) y Ford (1994) al referirse a los cruces aluvionales de culturas que generan una fermentación que entrevera solidaridades, diferencias, trances y fusiones de intereses. El resultado dista de ser parejo u homogéneo.

No obstante, en esa heterogeneidad Iberoamérica presenta cualidades que podrían motivar mayores acercamientos. Además de los lazos históricos (no exentos de conflictos, dramáticos en algunas etapas de la historia), de cierta proximidad cultural en el sentido antropológico del término y de dos idiomas vecinos como el español y el portugués que se practican con variantes en casi toda lberoamérica, en el campo de la comunicación los países del espacio iberoamericano tienen rasgos comunes. La predominancia de grupos como Telefónica y América Móvil, la estructuración conglomeral del sector infocomunicacional y la configuración asimétrica de los accesos a bienes y servicios de la comunicación y la cultura se cuentan como características distintivas de la mayoría de los países de lberoamérica. Los principales grupos que operan en este espacio son, en la mayoría de los casos, de origen iberoamericano, con la excepción de capitales estadounidenses en el caso de DirecTV (Becerra y Mastrini, 2016).

En efecto, la presencia de los mismos actores corporativos dominantes en el amplio sector infocomunicacional en muchos de los países iberoamericanos también conforma un sustrato común, en este caso de la estructuración económica de las comunicaciones, que imprime rasgos particulares. Las telecomunicaciones, el sector editorial de libros (aunque la circulación de materiales es restringida incluso en países limítrofes), parte de la producción cinematográfica y musical y, en menor medida, el segmento de contenidos y redes de televisión y radio se hallan dominados por grupos con proyección en todo el espacio o bien en gran parte de él. La economía del sector infocomunicacional cuenta con dos actores de origen de capitales iberoamericanos como pilares: Telefónica y América Móvil. Hay otros grupos que operan en el espacio que tienen sede en los propios países, aunque merece destacarse que en el segmento audiovisual ampliado (considerando televisión, cine y video), aunque aparenta ser paradójico, uno de los históricos rasgos comunes de lberoamérica es la exhibición de los mismos contenidos procedentes de EEUU. Tal es así que por ejemplo en tv abierta, aunque en países con fuerte industria de contenidos locales como Brasil, México, España, Argentina 0 Colombia los contenidos son mayormente de factura local, sin embargo el segundo origen en importancia de las producciones es EEUU.

Por su parte, la edición de diarios (impresos y online) es la única de las industrias culturales tradicionales que conserva una lógica de organización de capitales nacionales y caracteres idiosincráticos, con "campeones nacionales" y una serie de editores de periódicos sin proyección regional.

Como se mencionó, Telefónica, América Móvil, PRISA, AT\&T (tanto como operador de telefonía como de tv satelital, DTH), Viacom y Time/Warner son algunos de los conglomerados con presencia dominante en Iberoamérica. Si bien los dos primeros, principales operadores de telecomunicaciones iberoamericanos, tienen su matriz en España y México, en los últimos años fue incrementándose la participación de corporaciones de capitales de EEUU en dos niveles: por un lado, como inversiones en redes de telefonía (fija y móvil) y de tv paga; por otro lado, como intermediarios, catalogadores y prestadores de servicios digitales globales en Internet que absorben crecientes porciones de la renta de la economía digital (con Google, Facebook, Apple, Amazon, Spotify y Netflix, como mascarones de proa). En el segmento de comercio electrónico también se destaca a nivel latinoamericano la consolidación de Mercado Libre, plataforma con sede en Argentina.

Como buena parte de Occidente, Iberoamérica tuvo industrias culturales concentradas desde sus inicios. Los principales grupos del espacio iberoamericano fueron transformándose en grandes conglomerados que reúnen variadas actividades en su seno ya desde las últimas dos décadas del siglo XX. Antes, estos grupos habían sido empresas familiares con propensión a dominar un sector de actividad, una industria de medios, lo que se cristalizó como procesos de concentración horizontal a lo largo de las décadas de 1950 y 1960, fundamentalmente (ver Becerra y Mastrini, 2012). Hoy en día la lógica de acumulación de los principales grupos se basa no tanto en el poder de concentrar un mercado de medios o de cultura idustrializada específico (televisión, radio, prensa), lo que a su vez les otorgaba un estatuto de interlocución política privilegiado, sino en el ejercicio de posiciones dominantes en diferentes mercados en forma simultánea (Zallo, 2011). De allí su carácter conglomeral.

Además, las estrategias de las empresas de telecomunicaciones robustecieron la tendencia a una estructuración oligopólica de estas actividades, lo que acelera la tendencia a la concentración ya que la convergencia tecnológica entre medios, telecomunicaciones e Internet integra en las mismas plataformas de distribución a estos sectores, otrora separados. El comportamiento de América Móvil y Telefónica hacia nuevas actividades va en esa dirección convergente. Ambos grupos reforzaron su presencia en Iberoamérica en las últimas dos décadas y por ello hoy América Latina en particular presenta un panorama que, en lo que refiere a las telecomunicaciones (fijas y móviles), se asemeja al duopolio.

Como la convergencia digital torna difusas las barreras tradicionales entre medios y telecomunicaciones, lo que también impacta en la necesidad de revisar las vejas regulaciones por su obsolescencia en relación a cómo evolucionan las industrias que son objeto de regulación, los grandes grupos de comunicación en Iberoamérica se están reacomodando al nuevo entorno. Algunos de estos grupos aprovecharon la globalización diversificando sus intereses en otros países (fundamentalmente PRISA, Televisa, Cisneros y Globo entre los grupos multimedios y América Móvil y Telefónica en telecomunicaciones). A la vez, los grupos deben responder a los desafíos planteados tanto desde los sectores políticos que procuran redefinir el marco regulatorio, como desde las estrategias corporativas de las empresas telefónicas que se han tornado una competencia real a partir de la convergencia tecnológica y la integración de servicios (como el triple o cuádruple play).

Si se analizara la estructura, tamaño y ámbito de actividad de los principales grupos infocomunicacionales en Iberoamérica se apreciarían varios niveles: en primer lugar, el de las telefónicas líderes Telefónica y América Móvil, y de DirecTV como operador panamericano; en segundo lugar 
grupos multimedios como PRISA (España), Globo y Abril (Brasil), Televisa (México), Clarín (Argentina), Cisneros (Venezuela), los que superan con creces el umbral de ingresos de más de mil millones de dólares anuales; en tercer lugar el de grupos dominantes en países de economías grandes, como Colombia, o medianas, como Chile, y luego el resto (que a su vez, en un examen detallado, habilitaría la definición de subniveles dentro de este conjunto, que es vasto y heterogéneo). La disparidad en la consideración de la envergadura económica de los grupos que protagonizan la estructuración infocomunicacional iberoamericana se corresponde con las diferencias demográficas y económicas de este espacio que exhibe cuatro grandes mercados en información y comunicación como Brasil, México, España y Argentina, y entonces los grupos más importantes de estos países tengan un mayor despliegue. Pero también la habilidad de estos grupos para proyectarse regionalmente en EEUU (en el caso de grupos mexicanos) o exportar contenidos (caso Globo) también cuenta a la hora de analizar sus fortalezas.

La ausencia o erosión de actores públicos, que contrasta con la comparación que podría establecerse con países europeos no mediterráneos, es indicadora de la omnipresencia mediadora del mercado (Martín Barbero, 2007) tanto en actividades infocomunicacionales como de la cultura industrializada. Esta omnipresencia mediadora que troquela con lógica comercial la producción y circulación de contenidos y comunicaciones, sin embargo, no sutura la intensificación de los contactos y los conflictos inherente a la globalización y que expone a las culturas y a los procesos de comunicación a tensiones desconocidas.

En tanto, las iniciativas de política pública para atender a necesidades de cooperación en la producción, equipamiento, intercambio, circulación y/o exhibición de contenidos culturales en Iberoamérica no lograron en esencia compensar las asimetrías estructurales propias de la lógica de funcionamiento del sector infocomunicacional. Ejemplo de ello es el Programa Ibermedia. España fue, por lejos, el principal aportante de programas como Ibermedia destinados a la producción (y en particular, la coproducción) cinematográfica de ficción y documental iberoamericana, tanto en términos absolutos como relativos. Cerca del $45 \%$ de los fondos de lbermedia surgieron del tesoro español desde la creación de esta iniciativa en 1998, aunque en los últimos años se produjo una dramática reducción de su contribución (Camacho Chavez, 2016). Ibermedia es el programa institucional de cooperación más exitoso en el ámbito de la cultura iberoamericana, con más de 93 millones de dólares invertidos entre 1998 y 2017 y 1600 proyectos beneficiados (de ellos hubo 600 películas estrenadas), pero hay otras iniciativas como lbermúsicas (de aliento a la producción y circulación musical, creado en 2011) o lbermemorias (para preservar documentos sonoros y audiovisuales como patrimonio intangible de los países, creado en 2014) que acusan similares problemas con el añadido de que su financiamiento es menor y, por su más reciente creación, su consolidación dista de ser equivalente a la de Ibermedia.

La deriva del programa Ibermedia ilustra los claroscuros de la cooperación en producción de comunicación y cultura en el espacio iberoamericano y la tendencia a la cesión al mercado de las directrices de desarrollo de un campo que, sin orientación de políticas públicas, queda confinado a áreas específicas y legitimadas, sin externalidades al resto de las actividades.

\section{El campo académico: ¿por casa cómo andamos?}

La producción académica en comunicación es otra de las aristas que se exploran en el presente artículo como parte de la cooperación iberoamericana en cultura y comunicación. En el esfuerzo comprensivo de condiciones cambiantes, dispares y carentes de plena autonomía, resuena el legado de Giddens (1994), Bauman (1997) y Schlesinger (2011) sobre la configuración del campo académico como reflejo y como agencia: reflejo tanto de condiciones sociales, políticas y económicas como de agendas de otras latitudes que se acrecientan en contextos de semiperiferia (con el correlato de niveles de traducción y apropiación en otras temporalidades y sin los ecualizadores de aquellos países), pero también agencia, en el sentido del campo académico como laboratorio de ideas y proyectos. En este sentido, la producción académica en comunicación y cultura en lberoamérica muestra escenarios de correspondencia histórica entre temas y modas, condiciones materiales de circulación de revistas, libros, instituciones y condiciones institucionales en las últimas décadas con una tendencia creciente a la dispersión de esas variables que estructuran el campo.

Denis McQuail (2009) plantea una serie de variables que orientan el examen de la producción en comunicación en diferentes áreas geográficas y contextos históricos, en un artículo en que analiza escuelas europeas del campo disciplinar: organización del sistema de medios y actividades colindantes en cada país, tradición científica, lógica formativa de la educación superior (curriculum, escuelas, influencias y programas), política internacional de los países (que condiciona cercanías y vínculos estatales y comunitarios) y características lingüísticas, entre otras.

La formulación del campo es tardía en Iberoamérica respecto de otras latitudes. En la década de 1960 comienzan a sistematizarse estudios que más tarde se articularían como "comunicación", aunque hay importantes excepciones como los trabajos seminales de Antonio Pasquali (1963) en Venezuela o de Manuel Vázquez Montalbán (1963) en España. Mientras que España y Portugal sufrían las dictaduras de Franco y Salazar que limitaron el potencial desarrollo de las escuelas de periodismo y comunicación, en América Latina los veinte años previos a la institucionalización representado en la formalización curricular de licenciaturas en comunicación a mediados de la década de 1980 fueron prolíficos, con la producción intelectual de trabajos de comunicación política, de sociopolítica de las comunicaciones, de comunicación para el desarrollo y el cambio social, de semiótica y estudio de los discursos sociales, de economía de los medios y de estudios culturales. Autores como Armand Mattelart, Eliseo Verón, Luis Ramiro Beltrán, Diego Portales, Luis Gonzaga Motta, Oswaldo Capriles, Rafael Roncagliolo, Héctor Schmucler, Regina Festa, Juan Somavía o Jesús Martín Barbero (ver Barranquero, 2005; o Zarowsky, 2013) contribuyeron a cimentar un campo de estudios con impronta latinoamericana que constituye el acervo de perspectivas y temas sobre la que en la región se configuró el campo disciplinar.

En la composición del campo en Iberoamérica tuvieron especial participación organizaciones como CIESPAL, fundamentalmente en las décadas de 1960, 1970 y 1980 cuando aún no se había institucionalizado el campo. Esta primera etapa se caracteriza por la construcción de relaciones e intercambios fértiles, con figuras como la de Mattelart que operaron como "traductores culturales" (Zarowsky, 2013) y esfuerzos señeros como el de la revista "Comunicación y Cultura" que Mattelart codirigió junto a Héctor Schmucler.

A mediados de la década de 1980 la creación de licenciaturas en comunicación y periodismo en simultáneo abrió nuevos canales de diálogo que se consolidarían a través de encuentros periódicos de ALAIC, Felafacs, en determinadas secciones de IAMCR e ICA, y más recientemente en ECREA o Asociación Española de Investigación de la Comunicación (AE-IC).

Los vínculos transatlánticos transitaron por períodos diferentes y, mientras que la política exterior española tuvo interés especial por América Latina (desde 1990 hasta principios de Siglo XXI) hubo una intensificación de relaciones que se plasmaron en colaboraciones de equipos de 
investigación, formación posgradual de investigadores (en particular, latinoamericanos que estudiaron en universidades españolas), producción académica en revistas especializadas y libros conjuntos, en Portugal la lusofonía facilitó la mayor atención a las relaciones con Brasil. Esta segunda etapa de las relaciones de cooperación académica está signada por el proceso de institucionalización y correspondiente formalización de acuerdos y programas que consolidaron líneas de trabajo y acercaron equipos de investigación y docencia.

La sumisión de aspectos estratégicos de las políticas culturales, comunicacionales, informativas y de derechos de autor en los tratados bilaterales de "libre" comercio suscriptos entre países latinoamericanos y grandes potencias, así como el proceso de integración europea que tuvo creciente incidencia a partir de la creación de la Unión tras el Tratado de Maastricht (1992), fueron alejando o debilitando los lazos al interior del espacio iberoamericano en lo relativo a la producción académica común.

En la última década España y Portugal se hallan mucho más insertos en la dinámica europea, en buena medida potenciados por la integración curricular del Plan Bolonia (Espacio Europeo de Educación Superior). La mayor inserción y atención de España y Portugal a la Unión Europea repercute en el espacio iberoamericano, toda vez que la UE asigna escaso interés a Latinoamérica y la cooperación cultural entre ambas regiones, que era considerada "poco prioritaria" hace diez años (Bustamante, 2007), carece de políticas de fomento y de recursos asociados. Tampoco en América Latina se aprovechó el inédito crecimiento económico del lapso 2002-2012 y la contingencia de simultáneo acceso a la conducción estatal de gobiernos sumariamente (y no siempre correctamente) aludidos como "progresistas" para materializar los consabidos discursos a favor de la cooperación iberoamericana para institucionalizar y potenciar los espacios de proximidad cultural y comunicacional existentes.

Terminada la etapa de bonanza económica en la que hubo asignación de recursos para la cooperación en comunicación y cultura y en la formación de universitarios, el relevo parcial de las políticas públicas en la materia se ha financierizado y la tutela que otrora practicaban algunos estados hoy es ejercida por entidades bancarias, mayormente de España.

Con una tradición común iberoamericana y una dispersión reciente, la agenda de estudios en comunicación, comprende estudios sobre periodismo, mediatizaciones y semiótica, marketing y comunicación política, economía política de las industrias culturales y mediáticas, liderazgo, teorías (aunque no hay mucha producción teórica), historia, nuevos medios, recepción, framing y agenda, inmigraciones, identidades, sexualidades, representaciones, sociología de las redacciones, géneros y estudios de programación (y desprogramación), comunicación organizacional, procesos de integración regional (NAFTA, Mercosur, región andina) y comunicación intercultural. Si bien hoy la producción es dispar y dialoga con la que se realiza en otras partes del mundo, es posible distinguir las huellas de una tonalidad normativa y ensayística que tributa a la preocupación por articular, con urgencia, la producción intelectual y el cambio social (ver, al respecto, la interesante reflexión de Ferron y Guevara, 2017). Este aspecto es cardinal pues permite reflexionar sobre los límites del campo, las estrategias de construcción de legitimidad y prestigio, la cristalización institucional de dichas estrategias y sus formas de consagración, como se preguntaba Bourdieu (1988).

Si algo caracteriza la producción académica iberoamericana en comunicación y cultura ha sido su sincretismo teórico y metodológico, propio de la condición periférica en el mapa planetario, junto con un énfasis por la praxis, en muchos casos a expensas de la consistencia en los desarrollos teóricos y metodológicos propios. El registro informal de esta característica de la producción iberoamericana es visible fuera de la región, sea en publicaciones como en congresos y seminarios, y podría estimular la reflexión sobre los alcances y limitaciones de la contribución académica en el campo disciplinar así como renovadas estrategias de cooperación entre grupos e investigadores.

\section{A modo de conclusión: reflejo y agencia}

Con una estructuración de la base material de las industrias infocomunicacionales que exhibe procesos, actores y temporalidades comunes, la cooperación iberoamericana en comunicación y cultura ha sido sin embargo más bien irregular tanto en las políticas de producción de bienes y servicios simbólicos como en su potencial reflexivo en el campo académico, ejes que fueron abordados en el presente artículo. Asimismo, la estructuración material de un lenguaje común o familiar confiere a Iberoamérica características peculiares que colaboran en la producción de relaciones colaborativas y de cooperación.

Si bien los grupos protagonistas de la escena convergente entre telecomunicaciones y medios audiovisuales en lberoamérica tienen origen en el propio espacio geográfico, la propia transformación del ecosistema de las comunicaciones a nivel global inserta nuevos actores corporativos cuya incidencia es creciente y, en el cotidiano de millones de iberoamericanos, cardinal (Facebook y Google, centralmente). Además, la influencia de contenidos producidos en EEUU es parte de un lenguaje que, más allá de la importante producción nacional en varios de los países iberoamericanos, resulta una plataforma de circulación simbólica que sedimenta como sustrato común, con su secuela de códigos, géneros y sensibilidades.

En cuanto al abordaje de la cooperación en producción cultural, la historia reciente exhibe regularidades en la mirada que predomina en los apoyos públicos. Esa mirada, que en el análisis de Martín Barbero (2004) es "conservacionista", es reproductora de una perspectiva patrimonialista que se combina con el sostén de actividades legitimadas socialmente como el cine y la música, que son constructoras y difusoras de un star system iberoamericano que se consagra al interior de este ámbito geográfico-cultural y también en el consolidado segmento hispano en EEUU. En este sentido, las políticas estatales de cooperación en comuniación y cultura han puesto su acento en el aliento a la producción en algunos campos particulares (cine), mientras que fueron poco productivos en la generación de políticas y de reflexión crítica sobre la cesión de otros eslabones de la cadena de valor cultural y comunicacional en manos del mercado, validando así la mercantilización plena de la circulación cultural lo que, según Bustamante (2007), disminuyó su eficacia pública.

De este modo, mientras que la producción en comunicación y cultura en Iberoamérica tuvo un lapso de expansión durante la etapa 1990-2005, los ejemplos más estables de estrategias y prácticas de cooperación han sido gestionados por una lógica de mercantilización y administración privada o mixta. La dotación de recursos públicos ha sido espasmódica y evidencia una retirada en la última década.

Más allá de la agenda escueta de las políticas públicas, la cultura iberoamericana tiene al sincretismo como gen constitutivo. "La comunicación es vivida por las comunidades rurales o urbanas como la posibilidad de romper la exclusión", sugiere Martín Barbero (2007), lo que desborda los marcos de comprensión tradicionalistas, puesto que "hay en esas comunidades menos complacencia nostálgica con las tradiciones y una mayor conciencia de la indispensable reelaboración simbólica que exige la construcción de su propio futuro". 
Pero la cooperación no es sólo una alternativa más entre las opciones de política pública en el contexto actual, sino que en el marco de la globalización es, en la perspectiva iberoamericana, una estrategia defensiva que reconoce el valor cardinal de la comunicación y la cultura integrada en red frente a la fuerza desigual de los países iberoamericanos ante otras economías con alcance planetario. La emergencia de un espacio global y conectado que describe con precisión Castells (2009), paradójicamente no motiva a los gobiernos de los países latinoamericanos a aprovechar las redes históricas, culturales y vivenciales que los unen.

En cuanto al campo académico, en los últimos 50 años en que éste fue conformándose en lberoamérica, se distinguen tres etapas: la primera, fundacional, en la que se destaca el esfuerzo por establecer contactos y producciones aún en condiciones que dificultaban, en términos comparativos, su concreción; la segunda etapa de institucionalización con el correlato de sistematización de lazos y líneas de trabajo y coproducción al calor del apoyo de recursos estatales (sobre todo de la cooperación española entre 1990 y 2005, coincidente con un contexto de mayor presencia privada en América Latina a través de las privatizaciones de la década de 1990 y también con la profundización de políticas bilaterales de programas de formación e intercambio); y una tercera etapa, presente, de mayor dispersión de los lazos al interior del espacio iberoamericano en el campo de la comunicación y la cultura.

Esta dispersión merece analizarse también a la luz de la creciente influencia de lo que Moragas Spà llama las majors editoriales en comunicación (Sage, Routledge, Hampton Press, Blackwell, Oxford University Press) que uniformiza la literatura, en particular sus temas, estilos y tradiciones con anclaje casi excluyente en la cultura anglosajona. La mayor parte de la producción del campo académico en comunicación y cultura en Iberoamérica carece de una traducción al inglés, pero como hipótesis de cierre queda abierta la pregunta de si el problema de lenguaje no excede lo idiomático y se proyecta hacia la articulación entre teoría y empiria, es decir, hacia el laborioso proceso de realizar conceptualizaciones a partir de procesos específicos con coherencia metodológica.

Esta pregunta queda abierta porque al ser reflejo y agencia, el campo de la comunicación y la cultura en Iberoamérica cuenta con acreencias y con deudas que sólo la reflexión común y concertada podrá resolver a futuro.

\section{Referencias bibliográficas}

Barranquero, A. (2005). "Latinoamérica en la ruptura del paradigma de la comunicación para el desarrollo". En Punto Cero, vol. 10, n¹1, pp. 7-22. Disponible en: http://www.scielo.org.bo/scielo.php?script=sci_arttext\&pid=S1815-02762005000200002

Bauman, Z. (1997). Legisladores e Intérpretes. Sobre la modernidad, la posmodernidad y los intelectuales. Bernal: Universidad Nacional de Quilmes.

Becerra, M. y Mastrini, G. (2016). "Concentración y convergencia de medios en América Latina". En Ensambles, n³. Disponible en: http://www.revistaensambles.com.ar/ojs-2.4.1/index.php/ensambles/article/view/62/33

Becerra, M. y Mastrini, G. (2012). "Structure of the Info-Communications Sector in Latin America". En Bolaño, C., Mastrini, G. y Sierra, F. (eds.), Political Economy, Communication and Knowledge, pp. 221-247. Nueva York: Hampton Press.

Bourdieu, P. (1988). Cosas dichas. Buenos Aires: Gedisa.

Bustamante, E. (2007). "La urgente revisión de la cooperación iberoamericana en cultura-comunicación”. En Bustamante, E. (ed.), La cooperación cultura-comunicación en Iberoamérica. Madrid: Agencia Española de Cooperación Internacional.

Camacho Chavez, M. (2016). "El espacio audiovisual iberoamericano (EAl): un proyecto regional”. Tesis de Maestría en Cooperación Internacional para el Desarrollo defendida en el Instituto José María Luis Mora, mimeo, México. Disponible en: https://mora.repositorioinstitucional.mx/jspui/bitstream/1018/130/1/Mariana\%20Yurani $\% 20$ Camacho $\% 20 \mathrm{Ch} \%$ C3\%A1vez $\% 20$ _ $\% 20$ El $\% 20$ espacio\% 20audiovisual\%20iberoamericana.pdf

Castells, M. (2009). Comunicación y Poder. Madrid: Alianza.

Crusafón, C. (2011). "El espacio audiovisual euro - latinoamericano: el cine como eje central de la cooperación supranacional". En Revista Análisi, n0 40 , pp. $27-45$.

De Moragas Spá, M. (2011). Interpretar la comunicación. Estudios sobre medios en América y Europa. Barcelona: Gedisa.

Ferron, B. y Guevara, E. (2017). "Sociología política de la "comunicación para el cambio social": pistas para un cambio de enfoque". En Revista de Comunicación y Ciudadanía Digital COMMONS, vol. 6, n¹. Disponible en: http://revistas.uca.es/index.php/cayp/issue/view/254

Ford, A. (1994). Navegaciones. Comunicación, cultura y crisis. Buenos Aires: Amorrortu.

García Canclini, N. (2004). Diferentes, desiguales y desconectados: mapas de la interculturalidad. Barcelona: Gedisa.

Getino, O. (2004). "Las relaciones de América Latina y la UE en el sistema de industrias culturales". En Telos: cuadernos de Comunicación, Tecnología y Sociedad, nº 61, pp. 12-35.

Giddens, A. (1994). Modernidad e identidad del yo: el yo y la sociedad en la época contemporánea. Barcelona: Península. 
Martín Barbero, J. (2007). "La comunicación y la cultura en la cooperación para el desarrollo". En Bustamante, E. (ed.), La cooperación culturacomunicación en Iberoamérica. Madrid: Agencia Española de Cooperación Internacional.

Martín Barbero, J. (2004). "Políticas de interculturalidad". En Oficios Terrestres, n¹8, pp. 102-114.

McQuail, D. (2009), "Diversity and convergence in communication science: the idea of "national schools" in the European area". En Carpentier, N. et al. (eds.), Communication approaches to politics an ethics in Europe. Tartu: Tartu University Press.

Pasquali, A. (1963), Comunicación y cultura de masas. Caracas: Monte Ávila Editores.

Schlesinger, P. (2011). "Intelectuales y políticas culturales". En Albornoz, L. (comp.), Poder, medios, cultura: una mirada crítica desde la economía política de la comunicación, pp. 93-108. Buenos Aires: Paidós.

Vázquez Montalbán, M. (1963). Informe sobre la información. Barcelona: Fontanella.

Williams, R. (1980). The long revolution. London: Penguin.

Zallo, R. (2011). Estructuras de la comunicación y de la cultura. Políticas para la era digital. Barcelona: Gedisa.

Zarowsky, M. (2013). Del laboratorio chileno a la comunicación-mundo: un itinerario intelectual de Armand Mattelart. Buenos Aires: Biblos.

\section{Cómo citar este artículo en bibliografías - How to cite this article in bibliographies / references:}

BECERRA, M. (2017): "El campo de la comunicación y la cultura como reflejo y agencia en Iberoamérica". En Revista de la Asociación Española de Investigación de la Comunicación, vol. 4, número 8, pp. 10-16. 